\title{
Prevalence of early T-cell precursor acute lymphoblastic leukemia among immunophenotypically categorised acute T-cell lymphoblastic leukaemia cases
}

\author{
Monika Gupta, Pinki Devi*, Anjali Bishley, Sant Prakash Kataria, Rajeev Sen
}

Department of Pathology, Pt BD Sharma PGIMS, Rohtak, Haryana, India

Received: 10 August 2021

Revised: 07 September 2021

Accepted: 08 September 2021

\author{
*Correspondence: \\ Dr. Pinki Devi, \\ E-mail: drpinkitmr@gmail.com
}

Copyright: () the author(s), publisher and licensee Medip Academy. This is an open-access article distributed under the terms of the Creative Commons Attribution Non-Commercial License, which permits unrestricted non-commercial use, distribution, and reproduction in any medium, provided the original work is properly cited.

\begin{abstract}
Acute lymphoblastic leukaemia is the most common hematopoietic malignancy in childhood, comprising of B-cell lineage $(85 \%)$ and T-cell lineage (15\%). Recent studies have identified a subtype of T-cell acute lymphoblastic leukaemia (T-ALL) termed "early T-cell precursors (ETP)" recognised as a new provisional entity in 2016 update to the World Health Organization (WHO) classification of acute leukaemia, early T-cell precursor acute lymphoblastic leukemia (ETP-ALL) is characterized by a unique immunophenotype and genetic profile and its origin has been found to be from migration of cells from thymus to bone marrow. Hence, our study aims at reporting the prevalence of ETPALL among immunophenotypically categorised acute T-cell lymphoblastic leukaemia cases. Present work is a retrospective observation of acute T-cell lymphoblastic leukemias and reporting ETP-ALL cases seen during the period of over two years (from August 2018 to August 2020) received for flowcytometry in the department of Pathology, PGIMS, Rohtak, Haryana. Peripheral blood showed features of acute leukemia and immunophenotyping was performed. Fourteen cases were received for flowcytometry showing features of acute leukemia and immunophenotyping was performed revealing two ETP-ALL cases with positivity for cytCD3, CD7 (T-cell markers), HLA-DR, CD13 (myeloid marker-aberrant expression), sCD34, CD117 (stem cell markers), CD19 (B-cell marker) and dim expression of CD45. This study is a supportive data for immunophenotypic identification of ETP-ALL cases in centres where genetic study and other ancillary techniques are not available. It needs to be differentiated from non ETP-ALLs as this entity has been reported to show treatment failure with the treatment modalities for non ETP-ALLs.
\end{abstract}

Keywords: Early T-cell precursors, Acute lymphoblastic leukaemia, Flowcytometer

\section{INTRODUCTION}

Acute lymphoblastic leukaemia is the most common hematopoietic malignancy in childhood $\mathrm{S}$, comprising of B-cell lineage (85\%) and T-cell lineage (15\%). Recent studies have identified a subtype of $\mathrm{T}$-cell acute lymphoblastic leukaemia (T-ALL) termed "early T-cell precursors" recognised as a new provisional entity in 2016 update to the World Health Organization (WHO) classification of acute leukaemia, ETP-ALL is characterized by a unique immunophenotype and genetic profile and its origin has been found to be from migration of cells from thymus to bone marrow. ${ }^{1,2}$ Jinghui et al studying the genetic basis of this disease have found that ETP-ALL is distinct from the non ETP-ALL and represents a neoplasm of less mature hematopoietic progenitor or stem cell, with arrest at a very early maturational stage that retains the capacity for myeloid differentiation. ${ }^{3} \quad$ Immunophenotypically, it is characterized by the lack of T-lineage cell surface markers CD1a and CD8, weak or absent expression of CD5, positive for antigens such as CD7, CD2 and cCD3, 
aberrant expression of myeloid and hematopoietic stem cell markers such as CD13, CD33, 11b, HLA-DR, CD34, CD64 and CD117. MPO is negative and CD4 and CD123 may be positive in some cases. ${ }^{1,4,5}$

This is an uncommon neoplasm found in both children and adults, accounting for approximately $10-13 \%$ of cases of T-ALL in childhood and for $5-10 \%$ of cases of adult ALL. ${ }^{1}$ Being diagnosed as separate entity with limited data in adults, it needs to reported. ${ }^{6}$ Due to poor response of this entity towards lymphoid directed therapy, high proportion of remission failure and relapse, it's prognosis is poor. ${ }^{7-10}$ Since ETP-ALL have been found transcriptionally most related to hematopoietic stem cells and myeloid precursors, therapeutically drugs more active in myeloid tumors have been proposed in the literature. $^{8}$

As our country with such a huge population and hence high number of cases in proportion to the other developed countries, need to at least diagnose this entity immunophenotypically which has been mentioned as reliable and relatively cheaper technique for diagnosing as other genetic tests are not affordable.

Our study reports the two ETP-ALL cases among the immunophenotypically categorised acute T-cell lymphoblastic leukaemia cases.

\section{CASE SERIES}

Over the period of two years, we found 14 cases of acute lymphoblastic leukaemia. These constituted one female $(7.14 \%)$ and 13 males $(92.86 \%)$. Maximum number of acute lymphoblastic cases were in the age group of 10-19 years including both the ETP-ALL cases. One case was seen in the 0-9 years age group. Age groups of 20-29 years and 30-39 years constituted 3 cases each (Table 1).

Table 1: Age distribution.

\begin{tabular}{|lll|}
\hline S. no. & Age group (in years) & No. of patients \\
\hline $\mathbf{1}$ & $0-9$ & 01 \\
\hline $\mathbf{2}$ & $10-19$ & 07 \\
\hline $\mathbf{3}$ & $20-29$ & 03 \\
\hline $\mathbf{4}$ & $30-39$ & 03 \\
\hline $\mathbf{5}$ & $40-49$ & 00 \\
\hline $\mathbf{6}$ & $>50$ & 00 \\
\hline
\end{tabular}

Twelve cases $(85.17 \%)$ were of acute lymphoblastic leukaemia, 8 showing aberrancies and two cases (14.28\%) were identified as ETP-ALL (Table 2).

\section{Case 1}

A 29-year-old male patient presented to medicine OPD with fever and maleana. On physical examination patient had anemia and there was no organomegaly or lymphadenopathy. Complete blood count and peripheral blood film, revealed $64 \%$ blasts with anemia and thrombocytopenia (Table 3). Bone marrow aspiration performed, showed cellular BM with high blast counts.

Table 2: Aberrancies seen in T-cell ALL cases.

\begin{tabular}{|lll|}
\hline S. no. & No. of cases & Aberrancies \\
\hline $\mathbf{1}$ & 1 & CD 10, CD 13 \\
\hline $\mathbf{2}$ & 1 & CD 79a \\
\hline $\mathbf{3}$ & 2 & CD 10 \\
\hline $\mathbf{4}$ & 2 & CD 33 \\
\hline $\mathbf{5}$ & 2 & CD 117 \\
\hline $\mathbf{6}$ & 4 & No abberancies \\
\hline
\end{tabular}

Table 3: Complete blood count findings.

\begin{tabular}{|lll|}
\hline Parameter & Case 1 & Case 2 \\
\hline Hemoglobin (gm/d) & 7.3 & 12.7 \\
\hline $\begin{array}{l}\text { Total leucocyte count } \\
\text { (cells/cumm) }\end{array}$ & 22000 & 81000 \\
\hline Differential leucocyte count $\mathbf{( \% )}$ & \\
\hline Blasts & 64 & 85 \\
\hline Neutrophils & 04 & 01 \\
\hline Lymphocytes & 32 & 03 \\
\hline Monocytes & 00 & 00 \\
\hline Eosinophils & 00 & 01 \\
\hline Basophils & 00 & 00 \\
\hline $\begin{array}{l}\text { Platelet count } \\
\text { (lakhs/cumm) }\end{array}$ & 0.7 & 0.2 \\
\hline
\end{tabular}

\section{Case 2}

Blood sample of 15 years old male patient was received for complete blood count in the hematology department. Patient presented with fever and mild splenomegaly. Complete blood counts and peripheral blood findings showed $85 \%$ blasts, anemia and thrombocytopenia.

Bone marrow aspiration was performed in both the cases. These blasts in both the cases were small to intermediate sized, with high nucleo-cytoplasmic ratio, fine to clumped chromatin, inconspicuous nucleoli and scant basophilic cytoplasm. Immunophenotyping findings were consistent with ETP-ALL (Table 4).

Flowcytometry of case no 1 showed positivity for cytCD3, CD7 (T-cell markers), HLA-DR, CD13 (myeloid marker-aberrant expression), CD34, CD117 (stem cell markers), CD19 (B-cell marker) and dim expression of CD45. Immunophenotyping showed negative expression of CD1a, sCD3, CD5, CD2, CD4, CD8 and Tdt. Case no. 2 revealed $80 \%$ was positive for cytCD3, CD13, HLADR CD117 and CD19, negative for CD1a, CD2, sCD3, CD5, CD1a, CD4, CD8, CD10, CD20 and CD79a (Table 4 and Figure 1 and 2). 
Table 4 Findings of both the ETP-ALL cases.

\begin{tabular}{|lll|}
\hline Findings & Case 1 & Case 2 \\
\hline sCD3 & + & - \\
\hline CD5 & - & - \\
\hline cyt CD3 & + & + \\
\hline CD7 & + & - \\
\hline CD13 & + & + \\
\hline CD33 & + & - \\
\hline CD34 & + & + \\
\hline CD117 & + & + \\
\hline CD79a & - & - \\
\hline HLA DR & + & + \\
\hline MPO & - & - \\
\hline CD1a & - & - \\
\hline CD2 & - & - \\
\hline CD19 & + & + \\
\hline CD20 & - & - \\
\hline CD4 & - & - \\
\hline CD8 & - & - \\
\hline Tdt & + & - \\
\hline CD10 & - & - \\
\hline CD45 & Dim & Dim \\
\hline
\end{tabular}

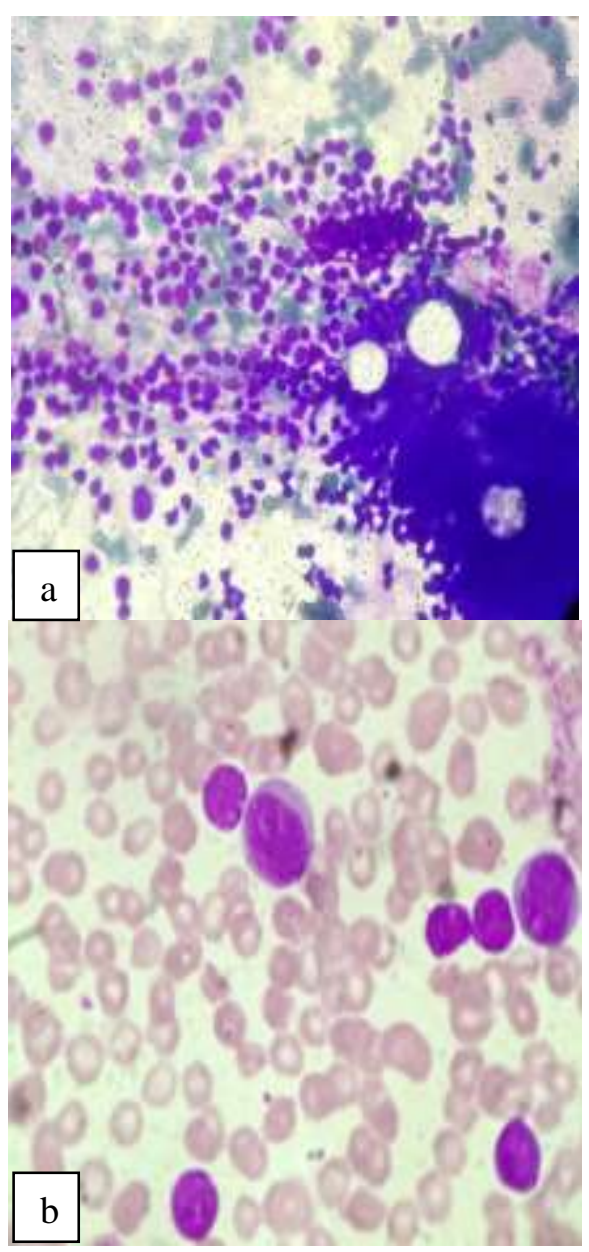

Figure 1 (a and b): Bone marrow aspiration smears showing blasts.

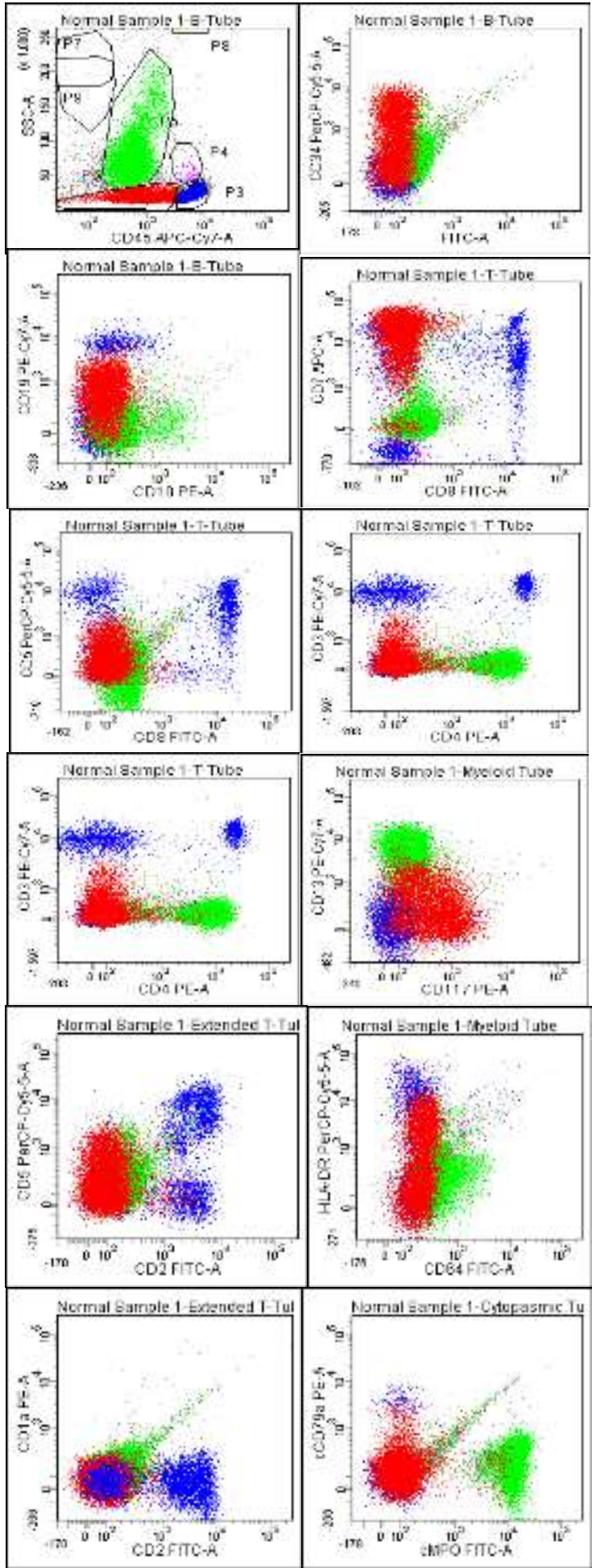

Figure 2: Flowcytometric findings for early T-cell precursor ALL blasts are; positive for CD34, CD19, CD7, CD117, HLA-DR, cCD3 and Dim CD45. Negative for CD10,CD8,CD5,CD4, sCd3, cCD79a, cMPO, CD1a ,CD64,CD2.

\section{DISCUSSION}

Early T-precursor acute lymphoblastic leukaemia/ lymphoma (ETP-ALL ) is a recently diagnosed subgroup 
of ALL which has been added as provisional entity in the 2016 update to WHO classification of acute leukaemia and has characteristic immunophenotypic and genetic profile. ${ }^{1}$ ETP-ALL is relatively uncommon as reported in many case studies and clinical trials it accounts to $5 \%$ to $36 \% .{ }^{11-13}$ Jain et al have reported $44(39.6 \%)$ cases of ETP-ALL and $67(60.4 \%)$ cases of non ETP-ALL cases in a study of 111 patients. ${ }^{14}$ Similarly, Khogeer et al have also found 33 (23.2\%) ETP-ALL and 109 (76.8\%) non ETP-ALL cases, similar findings were reported by Katharine et al where ETP-ALL cases accounted for $16 \%$ of all T-ALL cases, and Ma Meilin et al reported 12 cases (16.2\%) out of 74 cases, as ETP-ALL. ${ }^{10,15,16}$ These are close to the findings in our study of $14.28 \%$ ETP-ALL cases.

We have found $12(85.7 \%)$ non ETP-ALL cases.

Patients treated as per GRALL 2003 and 2005 treatment protocols for ETP-ALL and non ETP-ALL showed mainly young males (median age $=38.5$ years). ${ }^{17}$ Jain et al showed median age 29 years in ETP-ALL cases and median age of 31 years in thymic non ETP-ALL cases and 28 years in mature non ETP-ALL cases majority of the cases being males. ${ }^{14}$ Similarly, Khogeer et al also presented main presentation in males with ETP-ALL cases showing 40 years and non ETP-ALL cases showing 30 years as median age of presentation. ${ }^{15}$ These findings are in contrast to our study, where though we found majority of cases as young males, ETP-ALL group showed higher median age as compared to non ETP-ALL cases (median age $=18.5$ years). This can be explained by lesser number of ETP-ALL cases in our study.

Khogeer et al reported worse anaemia, higher blast counts in peripheral blood and bone marrow, similar findings were seen in study done by Jain et al. ${ }^{14,15}$ But our study again showed contrasting results, with non ETPALL showing worse anaemia. It can be attributed again to low case count. WBC counts in GRALL studies, study done by Jain et al and Khogeer et al showed lower counts in ETP-ALL than non ETP-ALL. ${ }^{14,15,17}$ Similar findings were confirmed in our study. Also, platelet counts study revealed the same trend as WBC counts in comparable studies.

Wang et al reported two cases ETP-ALL, presenting with fever and abnormal hemogram showing blasts, one case presented with lymphadenopathy. ${ }^{18}$ LBL cases in a study done by Jain et al presented lymphadenopathy as most common symptom followed by skin involvement, supraclavicular and tonsillar mass. ${ }^{14}$ Lymphadenopathy is most prominent presentation in our study mainly seen in non ETP-ALL cases and was not present in ETP-ALL cases. Hepatosplenomegaly was seen in ETP as well as non ETP-ALL cases. CNS involvement and mediastinal mass as mention in previous literature were not the features in our cases.
Flow cytometric findings of ETP-ALL cases reported by Wang et al were comparable to our cases, both showing positivity for $\mathrm{CD} 34, \mathrm{CD} 117, \mathrm{CD} 2, \mathrm{CD} 7$, one of the case was positive for CD33 (negative for CD13) and other was positive for CD13 (negative for CD33). Also HLADR was also found positive in only one of the case. Both cases were negative for CD5, CD1a, CD8, CD4, CD10, CD19, MPO. ${ }^{4}$ As compared to Khogeer et al CD1a was not included in all our cases, only done in two cases which had suspicion of ETP ALL. ${ }^{15}$ Also Jain et al has classified cases based on CD1A and CD3, into early thymic and mature. ${ }^{14}$ But we have segregated ETP ALL cases on the basis of immunophenotypic definition as mentioned in WHO.

\section{CONCLUSION}

We studied 14 cases among which two cases were immunophenotypically diagnosed as ETP-ALL as per 2016 revision of the WHO classification of myeloid neoplasm and leukaemia.

The main goal of the reporting these two cases among the total T-cell ALL cases is to highlight that the among patients with T-cell ALL cases which show better prognosis with conventional regimens, a significant number of cases (17-20\%) patients constitute ETP-ALL group which do not respond to these conventional regimens, hence need to be diagnosed correctly so that these patients can receive proper treatment on time which can further affect the outcome/prognosis. This study further supports the flowcytometry based diagnosis of this entity which makes it more convenient to be diagnosed, as flowcytometry is now a days available at many centres.

\section{ACKNOWLEDGEMENTS}

The authors thank the staff of flowcytometry laboratory at the Pt B. D. Sharma UHS, Rohtak Haryana.

Funding: No funding sources Conflict of interest: None declared

Ethical approval: Not required

\section{REFERENCES}

1. Arber DA, Orazi A, Hasserjian R, Thiele J, Borowitz MJ, Le Beau MM, et al. The 2016 revision to the World Health Organization classification of myeloid neoplasm and acute leukaemia. Blood. 2016;127:2391-405.

2. Shortman K, Wu L. Early $\mathrm{T}$ lymphocyte progenitors. Annu Rev Immunol. 1996;14:29-47.

3. Zhang J, Ding L, Holmfeldt L, Gang Wu, Heatley SL, Payne-Turner D, et al. The genetic basis of early T-cell precursor acute lymphoblastic leukaemia. Nature. 2012;481:157-63.

4. Wang P, Peng XX, Deng X, Gao L, Zhang X, Feng Y. Diagnostic challenges in T-lymphoblastic 
lymphoma, early T-cell precursor acute lymphoblastic leukemia or mixed phenotype acute leukemia: a case report. Medicine (Baltimore) 2018;97:e12743.

5. Chopra A, Bakhshi S, Pramanik SK, Pandey RM, Singh S, Gajendra S, et al. Immunophenotypic analysis of T-acute lymphoblastic leukemia. A CD5-based ETP-ALL perspective of non-ETP TALL. Eur J Haematol. 2014;92:211-8.

6. Castaneda Puglianini O, Papadantonakis N. Early precursor T-cell acute lymphoblastic leukaemia: current paradigms and evolving concepts. Ther Adv Hematol. 2020;11:2040620720929475.

7. Coustan SE, Mullighan CG, Onciu M, Behm FG, Raimondi SC, Pei D, et al. Early T-cell precursor leukaemia: a subtype of very high-risk acute lymphoblastic leukaemia. Lancet Oncol. 2009; 10:147-56.

8. Haydu JE, Ferrando AA. Early T-cell precursor acute lymphoblastic leukaemia (ETP T-ALL). Curr Opin Hematol. 2013;20:369-73.

9. Iqbal N, Sharma A, Raina V, Bakhshi S, Kumar R, Gajendra S. Poor response to standard chemotherapy in early T-precursor (ETP)-ALL: a subtype of T-ALL associated with unfavourable outcome: a brief report. Indian J Hematol Blood Transfus. 2014;30:215-8.

10. Ma M, Wang X, Tang J, Xue H, Chen J, Pan C, et al. Early T-cell precursor leukemia: a subtype of high risk childhood acute lymphoblastic leukemia Front Med. 2012;6(4):416-20.

11. Shah BD, Borate U, Kota VK, Zhang L, Peker D, Hathaway AR, et al. Multi-institution review of adult early T-cell precursor acute lymphoblastic leukemia/lymphoma (ETP-ALL). Blood 2015;126:3715.

12. Inukai $\mathrm{T}$, Kiyokawa N, Campana $\mathrm{D}$, Coustan-Smith E, Kikuchi A, Kobayashi M, et al. Clinical significance of early T-cell precursor acute lymphoblastic leukaemia: results of the Tokyo children's cancer study group study L99-15. Br J Haematol. 2012;156:358-65.

13. Allen A, Sireci A, Colovai A, Pinkney K, Sulis M, Bhagat G, et al. Early T-cell precursor leukaemia/lymphoma in adults and children. Leuk Res. 2013;37:1027-34.

14. Jain N, Lamb AV, O'Brien S, Ravandi F, Konopleva M, Jabbour E, et al. Early T-cell precursor acute lymphoblastic leukemia/lymphoma (ETP-ALL/LBL) in adolescents and adults: a highrisk subtype. Blood. 2016;127:1863-9.

15. Khogeer H, Rahman H, Jain N, Angelova EA, Yang $\mathrm{H}$, Quesada A, et al. Early $\mathrm{T}$ precursor acute lymphoblastic leukaemia/lymphoma shows differential immunophenotypic characteristics including frequent CD33 expression and in vitro response to targeted CD33 therapy. Br J Haematol. 2019;186:538-48.

16. Patrick K, Wade R, Goulden N, Mitchell C, Moorman AV, Rowntree C, et al. Characteristics and outcome of children and young adults with early T-precursor (ETP) ALL treated on UKALL 2003. Blood. 2013;122:58.

17. Bond J, Graux C, Lhermitte L, Lara D, Cluzeau T, Leguay $\mathrm{T}$, et al. Early response-based therapy stratification improves survival in adult early thymic precursor acute lymphoblastic leukaemia: a group for research on adult acute lymphoblastic leukaemia study. J Clin Oncol. 2017;35:2683-91.

18. Wang XX, Wu D, Zhang L. Clinical and molecular characterization of early $\mathrm{T}$-cell precursor acute lymphoblastic leukaemia: Two cases report and literature review. Medicine (Baltimore) 2018;97:e13856.

Cite this article as: Gupta M, Devi P, Bishley A, Kataria SP, Sen R. Prevalence of early T-cell precursor acute lymphoblastic leukemia among immunophenotypically categorised acute T-cell lymphoblastic leukaemia cases. Int J Res Med Sci 2021;9:3143-7. 